\title{
AN APPROACH ON DYNAMIC GEOSPAITAL INFORMATION SERVICE COMPOSITION BASED ON CONTEXT RELATIONSHIP
}

\author{
Dayu Cheng ${ }^{\mathrm{a}, \mathrm{b}^{*}}$, Faliang Wang ${ }^{\mathrm{b}}$ \\ ${ }^{a}$ China University of Mining and Technology, Xuzhou, China \\ ${ }^{\mathrm{b}}$ National Geomatics Center of China, Beijing, China \\ yuyumails@126.com
}

Commission VI, WG VI/1

KEY WORDS: Geospatial Information Service, Geo-collaboration Work System, Dynamic Composition, Context Relationship

\begin{abstract}
:
For the new demand of dynamic integration of spatial data, the model and collaboration of processing functions caused by characteristics of dynamic interaction and the participants random demand for information in geo-collaboration work system, for instance, during on-line consultation meeting, the paper presented an approach considering context dynamic service composition information. Firstly, the paper introduced the dividing method of context relation in consultation from the user and GI services, interaction between services and service perspective, constructed GI service context relation, and established GI service description model considering service's context relation based on OWL-S. For the problems above, the paper proposed an approach on GI service dynamic composition based on context relationship. Then, the paper provided a framework for GI services dynamic composition, and discussed every important component of framework. Finally, an experiment about checking illegal construction on boundary was implemented to illustrate the concepts and ideas discussed in the paper.
\end{abstract}

\section{INTRODUCTION}

Geo-collaboration work system is an information platform constructed by computer network, geographic information system technology etc, which provides online data sharing, information exchange and dynamic interaction for analyzing hot spot issues (ALAN M. MACEACHREN \& ISAAC BREWER, 2004). In recent years, with the emergence of SOC (Service-Oriented Computing), a new distributed computing model disposes the distributed storage data, models and processing functions as services through encapsulation by standard protocol. Consequently, the ideas and approaches for constructing loose coupling hot consultation system under the heterogeneous, opened environment are developed. However, during geo-collaboration environment, the users' demands for GI services are dynamic and random, so it need to be generating new GI service by combining existing services.

From the SOC point of view, GI service should be developed from static to dynamic. Currently, the portfolio of services is a more flexible dynamic business processes rather than static affair. No one denies that the main difficult for dynamic composition is the lack of computer-readable semantic information which is related to the semantic web and semantic web service starting point for research. However, the lack of the context relationship between GI services, which is built on interaction and transaction, is a deeper reason (CHEN Shi-Zhan \& FENG Zhi-Yong, 2010). Furthermore, studies about dynamic composition do not consider the information of customers' preferences, interaction habits, so those approaches are difficult to satisfy the dynamic interaction requirements. For the problems above, this paper proposes an approach on GI service dynamic composition based on context relationship by analyzing research status of GI service composition and considering a scene or participants preferences.

The remaining article is structured as follows: The problem of dynamic composition of GI services is introduced, and a solution method is proposed in Section 1. Section2 describes the context relationship about services and users. Section 3 presents the basic components and the architectural framework for composing GI services, and discusses steps of their implementation. Section 4 introduces an instance to verify the feasibility and effectiveness of the method. Finally, in Section 5 we conclude our work and briefly outline future research topics.

\section{GI SERVICE DESCRIPTION MODEL CONSIDERING CONTEXT RELATIONSHIP}

In general, any information used to characterize the situation of an entity can be defined as the context, which is widely accepted. Extending this context definition, we provide an context conception of GI service that the context includes not only the relationship of computational logic in GI services, but also the information of users' preferences, users' feedback for GI service implementation.

\subsection{Analysis of GI Service Relation}

In this article, GI service context addresses primarily the relationship among geospatial information services. The socalled service relationship is the binary relations, which is built on service interaction and transaction context, describes the different services between the functional and non-functional constraints (DEY A K et al, 2001). In the practical application

*Dayu Cheng. Email: yuyumails@126.com. Supported by the Project of the National Science@ Technology Pillar Program of china .No.2008BAK49B01. Project supported by the National Natural Science Foundation of China. No. 41001216 
with GI services, there are some correlations among web services, as subordinate to a geographic information application. For example, the slope analysis service, a kind of spatial geoprocessing service, corresponds between input and output data expressing the logical correlation for different data services. The relevance among services provides the basis reasoning rules for the dynamic services composition.

From the perspective of the GI services' description document, such as the OGC's GetCapabilities, GetMap, and GetFeatureInfo etc. (Joshua Lieberman et al, 2003), GI service can be abstracted to a conceptual model that is composed of three components, service, operation and parameter. Accordingly, the corresponding GI services relationship can be divided into three granularities, i.e., parameter-level, operationlevel and service-level relationship (CHEN Shi-Zhan \& FENG Zhi-Yong, 2010). The service-level relation describes the interaction and constraints between services, and is of greater significance for the practical application of business processes. Therefore, this paper presents the service relationship that mainly refers to the service-level hierarchy.

On the view of the GI services' function, it is not necessary to select the GI services with similar functions in the service composition. On the other hand, a basic peculiarity of SOC is to decompose problems into smaller, interrelated calculation logic unit (i.e., services), which determines those services that must have dependency relationship.

In addition, the spatial relationship, which is the primary characteristic to distinguish the geographic data from the general data, is defined as the relationship of the spatial characteristic among the geographic entities, such as the distance relationship, the direction relationship and the topological relationship, etc. It is also the basis of the spatial data to organize, query, analyze and deduce. As a network-level expression of the geographical data, GI services also inherit this characteristic.

From the point of relevant degree of GI services' function, the relationship of GI services can be classified both explicitly and implicitly. The explicit relationship indicates a direct correlation among services. The most typical is the IOPE (Input, Output, Precondition, Effect) model, describing the web services features and operating conditions, and stating the relationship between information converted (input, output) and the service execution state changes. In addition, there is a type of geographic information, which has no direct link between services and can only be reflected in a specific scenario or event- driven by some kind of calculation. This service is implicit association. For example, the GI services' direction relation can be reflected only through the different GI services in specific scenario.

Based on the above analysis, this article summarized seven types of GI service relationships into three categories and shown in Table 1, and gives a definition of the ontology of GI service relationships, as shown below:

Definition 1 (GI service relationship ontology) It is composed of two-tuple, GIRS $=(S, R)$, where $S$ is the set of GI services, which is expressed as $S=\left\{W S_{1}, W S_{2}, \ldots, W S_{n}\right\}$.In which, $\left\langle W S_{i}, W S_{j}\right\rangle$ denotes that $W S_{i}$ is directly or indirectly associated with $W S_{j}$, and $R$ is the specific mapping between the services, its members have the following relationship: $\rho=f(d)$ or $\rho^{*}=f(d)$, where $d$ is the service description document of $W S_{j}$. Specifically, the sign of $\rho$ is described as properties in a document properties, and $f$ denotes the mapping rules.

\begin{tabular}{|l|l|l|l|}
\hline Relation type & $\begin{array}{l}\text { Relation } \\
\text { Name }\end{array}$ & $\begin{array}{l}\text { Relation } \\
\text { Intensity }\end{array}$ & $\begin{array}{l}\text { Attribute } \\
\text { Type }\end{array}$ \\
\hline $\begin{array}{l}\text { Spatial } \\
\text { Relationship }\end{array}$ & Topological & implicit & static \\
& Distance & implicit & static \\
& Position & implicit & static \\
$\begin{array}{l}\text { Business } \\
\text { Relationship }\end{array}$ & Dependency & explicit & static \\
& Complement & explicit & static \\
$\begin{array}{l}\text { Function } \\
\text { Relationship }\end{array}$ & Precursor & explicit & dynamic \\
& Successor & explicit & dynamic \\
\hline
\end{tabular}

Table 1. Relationships of GI Services

\subsection{User Context}

User context provides users' personalized information and performed services in the business process. It is fact component for reasoning and as the basic restriction for latter GI services. User context includes personalized profile, user location information, etc.

Definition 2 (user context) It is composed of fourtuple $U$-Context $=\{$ userID, preSList, pres, $F\}$, where user ID is the user's identifier, and PreSList is a set of GI services that have performed by user in the business process. Every GI service in set of PreSList is described by $S$. It is constructed by two-tuple, $S=\{$ SName, result $\}$. SName is GI service's name, and result indicates service state whose value is either true or false. Pres is a set of GI services in current session. F is a set of assert which is fact base for reasoning and includes user personalized profile.

\subsection{Service Description Model}

The traditional description model of GI services mostly concerns about the interface properties of the service itself, such as W3C's WSDL and OGC's spatial service description model etc. This kind of description model based on keyword lacks GI services' semantic information and cannot realize automatic discovery and dynamic composition of services. Furthermore, the current languages for describing semantic web services is still based on the interface description and only attends services' own behavior. For instance, the OWL-S abstract model describes the input, output, front and results (IOPE) of services, but it does not describe the relationship between the input and the output. Therefore, in order to use the context 
relationship to achieve the dynamic composition of GI services, we should firstly extend the current GI description model.

The GI service description model considering the service relationship should depict service information accurately in terms of both services' syntax factor and semantic factor, and provides a basis for automatic discovery and customization of GI service. Specifically, the service description model in this article must be able to describe the following information (XIE Dan et al, 2008):

1. Physical information: It describes a GI service information about URL address, and the function interface parameters which includes parameter' format, number, order and other information for input and output data.

2. Performance information: It indicates QoS(Quality of Service) factor and other non-functional performance indicators of GI services, such as the information about the duration needed for completing the service, the cost of the service, the service level the security etc.

3. Semantic information: It describes a functional semantics of GI service for understanding and handling by computer. In order to support the semantic description, we should unify domain knowledge through building application ontology repository, and provide the semantic basis for the understanding and interaction among different entities.

4. Context information: It describes the condition of the cooperation between the service and other services. Through recording the successful assembled cases, it provides the further support for the service discovery and dynamic composition.

For realizing the above information in service description model, we extend the OWL-S model, and add four-level semantic information about the function, interface, implementation and QoS. The detail semantic information is defined as follows:

1. Function semantic: It includes the domain classification of services and GI services' ability.

2. Interface semantics: It describes the operation and the message of the semantic Web services to facilitate the automatic discovery and composition about the service.

3. Execution semantics: It describes the pre-operation and the post-operation of the semantic Web services to facilitate the composition and dynamic configuration of the service.

4. QoS semantics: it describes the service quality of the semantic Web services to facilitate the improvement of the quality of the service discovery.

The extend OWL-S Model is shown in Figure 1.

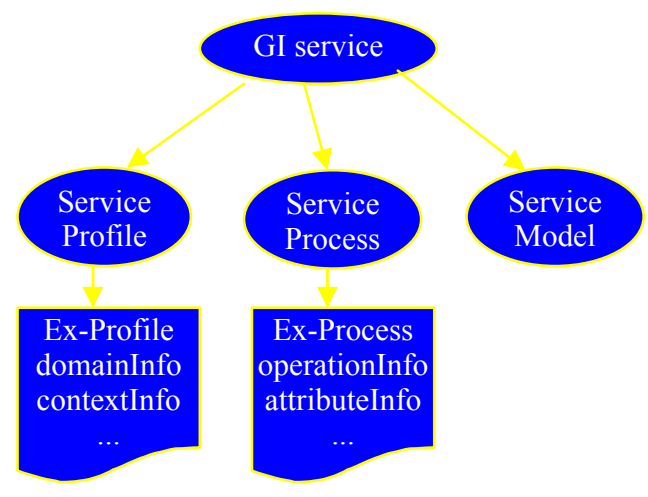

Figure 1 Extend OWL-S model for GI services

\section{FRAMEWORK FOR GI SERVICES DYNAMIC COMPOSTION}

In this section, we introduce the dynamic service composition framework for GI services, describe the service description model considering context relationship, and provide a scenario for reasoning based on service relationships.

\subsection{Dynamic Composition Framework}

On the global view for analyzing and constructing user's need, the GI service of user's request can be abstracted into business process composed of several service nodes. Each node may be an atomic service or service group, which consists of several services, and through user interaction to build and assemble service nodes to form a conceptual model for composition service.

At the same time, we import the context concept, and convert the user's individual demand into constraint condition for compositing by defining the context relationship of the GI services and users' context information. According to the business process and constraint of the context relationship, a mapping from conception model of composition service to actual GI service would be built. When composition service is implemented, the actual GI service should be bound dynamically, and an executable GI service chain is built. Figure 1 illustrates the technical framework.

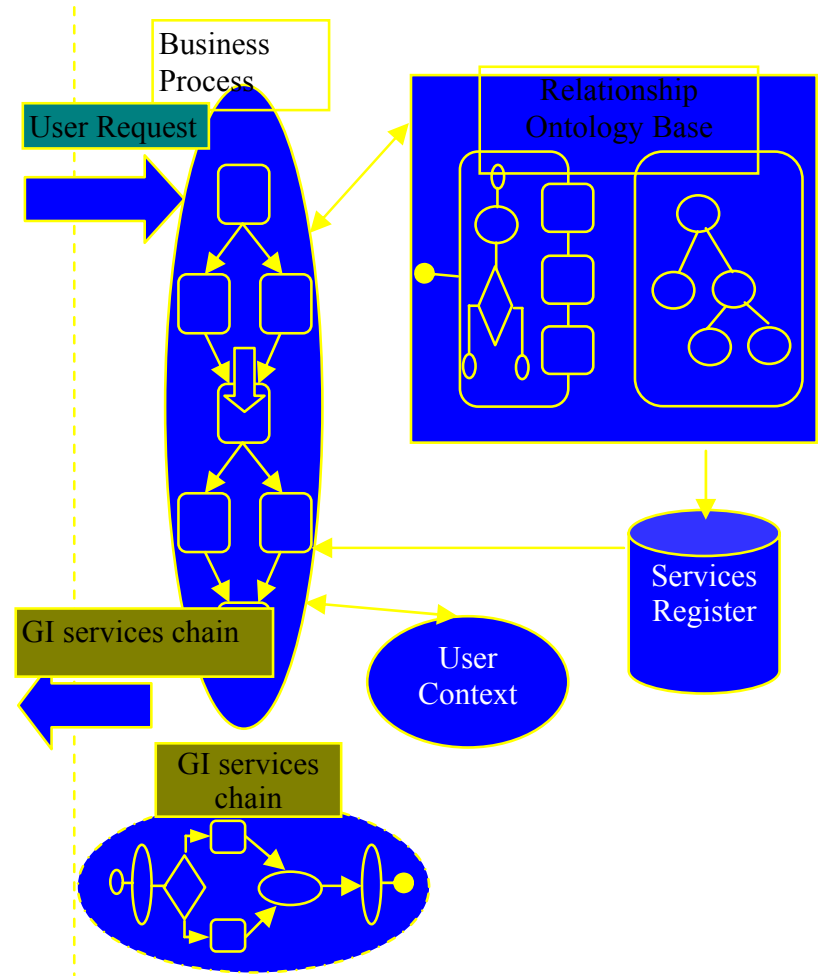

Figure 2. The GI service framework considering the context

The dynamic composition based on context relationship adopts the strategy of trading space for time. It should mine and store the service relationships in advance, and build some service nodes templates on the level of large granularities. For this approach, the ontology of GI service context is the basis of the 
dynamic reasoning for GI services. When building composition conceptual model and mapping from conceptual model to actual GI service, we should fully utilize the service context relationship and the method of relationship deduction. We also import the structural characteristics and the relationship reasoning of the OGC service chain into the service composition algorithm based on semantic and AI deduction.

\subsection{Design for Reference}

In the framework of the dynamic service composition, how to do reasoning based on the context relationship is a difficult problem and needs to be solved. The reasoning of the service relationship ontology is mainly based on the method of TBOX (assertions on concepts) and ABOX (assertions on individuals), and it can achieve the deduction of the concept-to-concept and concept-to-entity. However, this method is far from meeting demands for the deduction about the dynamic service composition oriented business processes. Therefore, this paper introduces SWRL (semantic web rule language) (W3C,2005, Yue, $\mathrm{P}$, et al, 2007), which is a representation language based on the ontology rule, and constructs the knowledge rule base. The ontology reasoning can make the service relationship ontology having better hierarchy structure, and ensure no conflicts among the GI service ontology. SWRL is mainly to achieve the deduction on the combination between the ontology knowledge and rule. The inference design is primarily divided into three parts:

1. The establishment of the ontology repository based on OWL service relationship and rules library based on SWRL. The dynamic composition considering the context relationship adopts the "trade space for time" strategy, and builds the service relationship library and deduction rules in advance. The establishment of the ontology and rules library can be manually built by expert advice, or automatically built through mining and constructing. This paper adopts non-automatic methods to build them.

2. Selecting the appropriate rule engine and converting the OWL ontology and SWRL rules into the acceptable format for the selected rule engine. As the ordinary rule engine cannot identify the OWL ontology and SWRL rules at the same time, they should be translated into appropriate formats. The paper uses the Jess rule engine, which provides a conversion method for OWL and SWRL formats.

3. Importing knowledge and rules into rule engine for inference. Through the format conversion as in the above step, the knowledge base and rule base are imported into the Jess rule engine and then computed. When the computation is finished, the Jess rule engine would return the final deduction result as the Jess knowledge format. To implement the deduction result interaction between engine and application, Jess API is Taken.

\section{EXPERIMENT}

To verify the practical value of the above method, we tested it by the instance of checking illegal building around land use boundary. The specific process is described as follows. On-site inspectors return on-site information (names or location coordinates, boundary coordinate information surrounding buildings). Boundary management users access the returned geographic information about the boundary in the field (such as those containing boundary information maps, image information, etc.) In accordance with the provisions of boundary regulations that it cannot have a permanent building materials within 10 meters around the boundary, a $10 \mathrm{~m}$ buffer zone is created around the analysis to determine the result by analysis the above result and building layer overlay.

For realizing GI service of checking illegal builds, firstly, user designed the business process model based on GI service temple which is looked as node of business process and prebuilt by experts in some domain. The business model of instance is shown as Figure3. Then, according to businessdriven and services' relationship reasoning, a mapping from actual GI services to the nodes of business process was automatically constructed. Where two ways for automatic reasoning, one is using services relationships, such as due to the precursor and successor relationship of buffer service discover the input GI service and output services, and other way is construct rule for reasoning. For example, for getting land use boundary information intelligent, a Geocoding service rule should be built by SWRL. The rule is as shown.R1:User(?x,?y)$\vee$ User(?addressName) $\Rightarrow$ Geocoding(?x,?y) $\vee$ Geocoding(? addressName). Through above reasoning with the service relationship, refine the business logic model. The detailed results are shown in Figure 4.

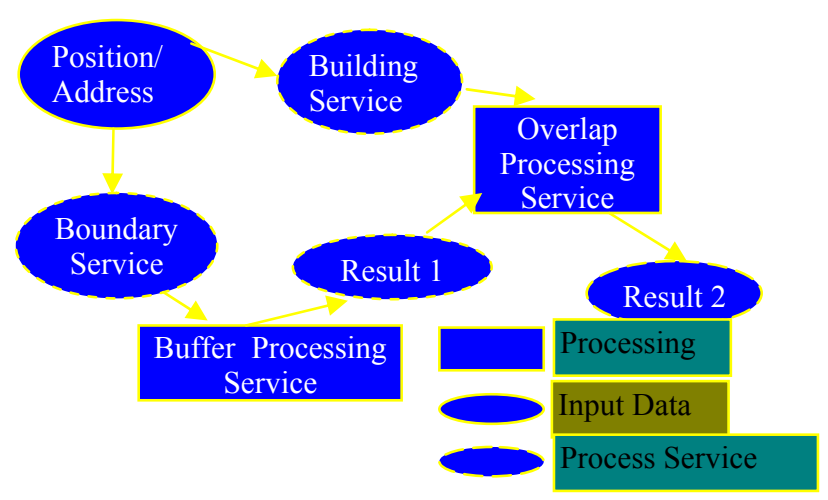

Figure 3. Conceptual business model of composition

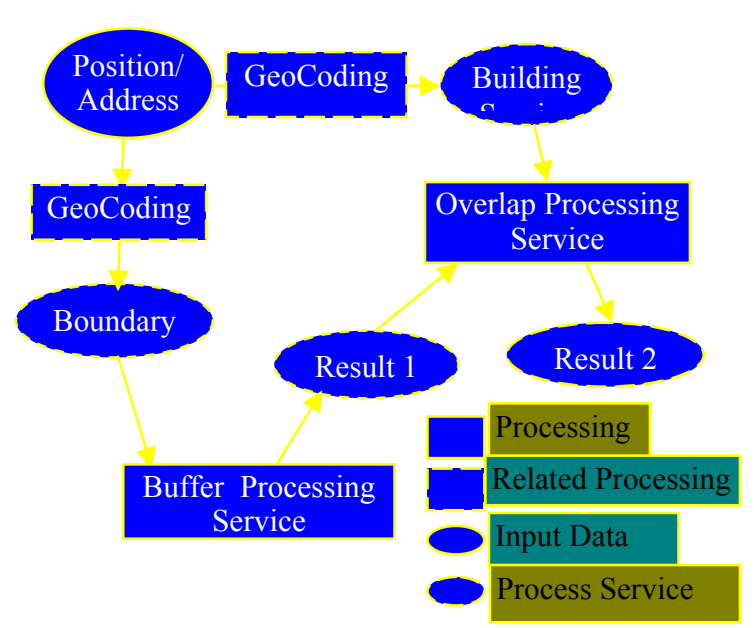

Figure 4. Business detail model of composition

Finally, when composition service running, the actual GI services are executed. 


\section{CONCLUSIONS AND RECOMMENDATIONS}

The problem of dynamic composition of GI service is a new and very important issue in the field of spatial information. Currently, related studies are short of considering the context relationship between GI services In this article, we present a promising solution for dynamic composition of GIS services based on the context relationships. To achieve this, we defined context information related GI services and context relationship between user and GI service in terms of spatial data characteristics, business process and service function. Based on the context relationship of GI services, we introduced a framework for dynamic composition of GI services. We also studied the GI service description models, considering context relationship by OWL-S, and discussed the important component in framework in detailed. To prove our idea, an experiment about checking illegal construction boundary was implemented to illustrate the concepts and ideas discussed in the paper.

The paper puts forward a basic idea on dynamic portfolio based on context relationships, and there is much work which requires further study, such as the improvement of the ontology of the service relationship and the optimization of the deduction rules. We plan to research these in future.

\section{REFERENCES AND BIBLIOGRAPHY}

ALAN M. MACEACHREN and ISAAC BREWER,2004, Developing a conceptual framework for visually-enabled geocollaboration, INT.J.Geographical Informatin Science, 18(1):pp. 1-34

CHEN Shi-Zhan, FENG Zhi-Yong, WANG Hui, 2010, Service relations and its application in services-oriented computing, Chinese journal of computers,33(11),pp. 2068-2083.

DEY A K, ABOWD G D, SALBER D, 2001, A conceptual framework and a toolkit for supporting the rapid prototyping of context-aware applications. Human-Computer Interaction Journal, 2001, 16(1).

Joshua Lieberman, Syncline Inc, 2003, OpenGIS Discussion Papers: OpenGIS Web Services Architecture.

W3C. 2001, Web Services Description Language (WSDL) 1.1, http://www.w3.org/TR/wsdl

XIE Dan,YING Shi,CAO Honghua, et al.2008, Resource description framework for service RDF4S based on semantics, 54(1), pp. 71 76.

W3C.2005, A proposal for a SWRL extension towards firstorder logic, http://www.w3.org/Submission/2005/SUBMSWRL-FOL-20050411/

Yue, P, et al. 2007, Semantics-based automatic composition of geospatial web service chains, Computers \& Geosciences, 33, pp.649-665. 